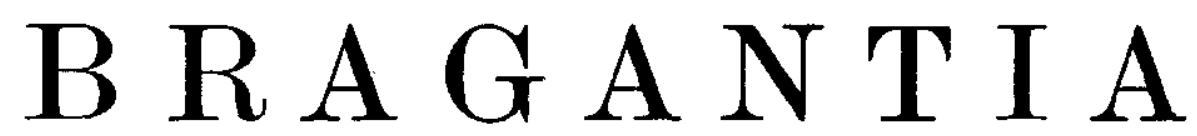

Revista Cientifica do Instituto Agronômico do Estado de Säo Paulo

Vol. 33

Campinas, julho de 1974

N. ${ }^{\circ} 7$

\title{
COMPORTAMENTO DE CULTIVARES DE TRIGO EM SOLOS DE VĀRZEA DO ESTADO DE SÃO PAULO $(1,2)$
}

Carlos Eduardo de Oliveira Camargo $\left({ }^{3}\right)$, Seçâo de Arroz e Cereais de Inverno, Túlio Ribeiro Rocha, Estação Experimental de Mococa, Instituto Agronômico, NoRberto Leite, Serviço do Vale do Paraiba, Departamento de Aguas e Energia Elétrica, Eduardo Issa $\left(^{3}\right)$, Seção de Doenças das Plantas Alimenticias Básicas $e$ Olericolas, Instituto Biológico

\section{SINOPS E}

Foram realizados três ensaios de competição de cultivares de trigo (Triticum aestivum L.) em diferentes várzeas do Estado de São Paulo.

Os cultivares IAC-5, S-12, BH-1146 e IRN-526-63 foram superiores na Estação Experimental de Mococa; os cultivares S-12 e Pitic-62 mostraram bom comportamento na várzea da Fazenda Jamic, município de Guatapará; e os cultivares IRN-152-63, Sonora-63, LA-1434 e IRN-526-63 apresentaram boa adaptação às condições do Campo de Pesquisas do Serviço do Vale do Paraíba, em Pindamonhangaba.

Além dos dados de produção foram também avaliados os seguintes caracteres: altura das plantas, ciclo, resistência ao acamamento e às doenças, comprimento das espigas, número médio de espiguetas por espiga, número médio de grāos por espiga e por espigueta.

\section{1 - INTRODUÇAO}

A cultura do trigo vem-se expandindo em vários paises, devido ao uso de cultivares mexicanos que foram adaptados a condiçōes ecológicas diversas e asseguram o sucesso da triticultura com irrigação aliada a altas doses de adubação e moderna tecnologia agrícola.

(1) Recebido para publicasão em 17 de abril de 1974.

(2) Os autores agradecem a colaboraçăo do Eng. Agr.o Derly Machado de Souza, na elaboração deste trabalho. 
Borlaug (1) mostra que a área semeada com cultivares mexicanos no Paquistão, em 1964, era de quatro hectares, e em 1967, de 1200000 hectares; na India, em 1964, foram plantados seis hectares, e em 1967, 2400000 hectares. Esses dados indicam o grande progresso da triticultura mundial, provocando a revolução verde em paises tradicionais importadores desse cereal.

Estudos realizados na india (5) mostraram que os cultivares de trigo anãos e semi-anãos reagiram, em condição de irrigação, a todos os níveis de nitrogênio (50-100-150 kg/ha), embora as reações tenham sido melhores nas doses mais elevadas. Por outro lado os cultivares sem fatores genéticos para nanismo acamaram muito quando adubados com $100 \mathrm{~kg}$ de $\mathrm{N} / \mathrm{ha}$ e obtiveram um aumento de rendimento apenas até à dose de $50 \mathrm{~kg} / \mathrm{ha}$. Dessa forma, mesmo em condições de elevada adubação, o trigo anão pode beneficiar-se da irrigação, sem acamar (5).

Os cultivares de trigo apresentam-se divididos em duas classes, com relação ao diferencial de tolerância à solução de alumínio (6), uma em que as plantas são severamente prejudicadas e sensiveis ao aluminio $\left(\mathrm{Al}^{3+}\right)$ enquanto em outra as plantas não são afetadas e são tolerantes.

No Estado de São Paulo (4) o trigo é plantado, na sua grande totalidade, na Região Sul e no Vale do Rio Paranapanema, mas existem grandes possibilidades de ser cultivado em várzeas, em outras regiões do Estado, bem drenadas, com facilidade de irrigação, porém normalmente com presença de $\mathrm{Al}^{3+}$, fazendo rotação com a cultura do arroz, que ocupa grandes ăreas nessas condições.

O presente artigo apresenta os resultados obtidos no ano de 1971, em Ensaios Regionais Paulistas de Cultivares de Trigo, conduzidos em três diferentes várzeas do Estado de São Paulo. Os cultivares foram eleitos como os mais promissores para condições de várzea, em estudos anteriores (3).

Para obter o máximo de informações sobre os cultivares, realizaram-se estudos sobre as características de produção, ciclo, altura, acamamento, resistência às doenças, tolerância ao alumínio $\left(\mathrm{Al}^{3+}\right)$, comprimento médio das espigas, número médio de grãos por espiga, número de espiguetas por espiga, número médio de grãos por espigueta e peso de 1000 grãos. 


\section{2 - MATERIAIS E MÉTODOS}

A relação e a origem dos dez cultivares de trigo estudados encontram-se a seguir.

IAC-s ou Maringá - Oriundo da progênie 16494, que proveio de seleção do híbrido entre a progênie 7124 e o cultivar Polissu $\left(\mathrm{PG}_{1}\right)$, em 1956, na Estação Experimental de Capão Bonito. A 7124 resultou de seleção do híbrido entre os cultivares Frontana e Kenya, em 1951.

S-12 - Introduzido pelo Instituto Biológico, oriundo da Secretaria da Agricultura do Estado do Rio Grande do Sul, criado na Estação Experimental de Júlio de Castilhos e obtido por seleção do cruzamento do hibrido entre os cultivares Veranópolis e Mayo 54 e o Veranópolis.

IAS-49 ou Pioneíro - Introduzido pelo Instituto Agronómico, Campinas, São Paulo, no ano de 1964, sob n.॰ 29816, originário do Instituto de Pesquisas e. Experimentação Agropecuária do Sul, Pelotas, Rio Grandẹ do Sul.

BH-1146 - Selecionado no Instituto Agronômico de Minas Gerais, Belo Horizonte, e proveniente do cruzamento Ponta Grossa IX Fronteira, hibrido esse que foi cruzado com o cultivar Mentana. O cultivar BH-1146 foi utilizado como controle, por ser o mais cultivado comercialmente na ocasião no Estado de São Paulo.

Sonora-63, IRN-526-63, LA-1491, LA-1434, IRN-152-63 e Pitic-62 foram selecionados no Centro Internacional de Melhoramento de Milho e Trigo (CIMMYT), México, e introduzidos através do Ensaio Internacional de Ferrugem: de Trigos de Primavera, do ano de 1963, pela Seção de Doenças das Plantas Alimenticias Básicas e Olericolas, do Instituto Biológico do Estado de São Paulo.

Nos ensaios de cultivares de trigo em condição de várzea utilizou-se o delineamento estatístico reticulado balanceado $3 \times 3$, com controle em cada sub-bloco, com quatro repetiçôes por local.

Foram retiradas amostras compostas dos solos dos locais estudados, anteriormente ao plantio, cujos resultados analíticos (4) foram os seguintes:

\section{Ensaios de}

\begin{tabular}{|c|c|c|c|}
\hline DETERMINAÇŌES & Mococa & Guataparó & Pindamonhangaba \\
\hline $\mathrm{pH}$ int & 5,10 & 5,60 & 5,40 \\
\hline $\mathrm{C} \% \quad \ldots \ldots \ldots \ldots$ & 1,50 & 290 & 6,20 \\
\hline$K^{+}(5)$ & 0,20 & 0,26 & 0,22 \\
\hline $\mathrm{Ca}^{2+}+\mathrm{Mg}^{2+}\left({ }^{5}\right\rangle \ldots$ & 3,40 & 3,80 & 4,80 \\
\hline $\mathrm{PO}_{4}^{3-}{ }^{3-}(6) \quad \ldots \ldots \ldots$ & 0,11 & 0,08 & 0,35 \\
\hline $\mathrm{Al}^{3+}{ }^{(5)} \quad \ldots \ldots \ldots \cdots$ & 1,00 & - & 0,70 \\
\hline
\end{tabular}

(4) Análise efetuada na seçăo de Fertllidade do Solo, Instituto Agronomico.

(5) e.mg/100 $\mathrm{ml}$ de solo. Teores trocáveis.

(8) e.mg/100 $\mathrm{ml}$ de solo. Teor solúvel erm $\mathrm{H}_{2} \mathrm{SO}, 0,05 \mathrm{~N}$. 
No ano de 1971, o Ensaio Regional Paulista de cultivares de trigo en condição de várzea foi semeado no dia 30 de abril, na Estação Experimental de Mococa; no dia 10 de maio, na fazenda de propriedade da Companhia Colonizadora Jamic, município de Guatapará; e no dia 12 de maio, no Campo de Pesquisas do Serviço do Vale do Paraiba, Departamento de Aguas e Energia Elétrica, municipio de Pindamonhangaba.

As parcelas foram constituídas de sete linhas de cinco metros de comprimento, espaçadas de 0,20 metro.

A semeadura foi feita na base de 40 sementes úteis por metro de sulco, equivalendo a 1400 sementes úteis por parcela, e por ocasião da colheita foram colhidas as cinco linhas centrais de cada parcela, deixando as demais como bordadura, portanto com uma área útil de $5 \mathrm{~m}^{2}$.

A adubação utilizada foi de $60 \mathrm{~kg}$ de nitrogênio, $90 \mathrm{~kg}$ de $\mathrm{P}_{2} \mathrm{O}_{5}$ e $20 \mathrm{~kg}$ de $\mathrm{K}_{2} \mathrm{O}$ por hectare, nas formas de sulfato de amônio (20\% de $\mathrm{N}$ ), superfosfato simples $\left(20 \%\right.$ de $\left.\mathrm{P}_{2} \mathrm{O}_{5}\right)$ e cloreto de potássio $\left(60 \%\right.$ de $\left.\mathrm{K}_{2} \mathrm{O}\right)$.

O modo de aplicação dos adubos obedeceu ao seguinte critério: $1 / 5$ do nitrogênio e todo o fósforo e potássio forarn aplicados no sulco de plantio, e os restantes $4 / 5$ do nitrogênio aplicados 30-40 dias após a germinação.

A descrição detalhada dos caracteres estudados, incluindo-se os métodos de determinação, foram citados em outro trabalho (4).

\section{3 - RESULTADOS E DISCUSSÃO}

As produções médias dos experimentos e média geral, em $\mathrm{kg} / \mathrm{ha}$, acham-se no quadro 1 .

Aplicando o teste de Tukey, ao nivel de 5\%, na comparação das médias do ensaio de Mococa, verificou-se que os cultivares IAC-5, S-12 e BH-1146 foram superiores em produção aos cultivares LA-1434, Sonora-63, Pioneiro, LA-1491 e Pitic-62. 
Quadro 1. - Produções médias de grãos dos Ensaios Regionais Paulistas de Cultivares de Trigo, instalados no ano de 1971 na Estação Experimental de Mococa, na Companhia Colonizadora Jamic, em Guatapará e no Campo de Pesquisas, em Pindamonhangaba

\begin{tabular}{|c|c|c|c|c|}
\hline Cultivar & Mococa & Guatapará & Pindamonhangaba & Média \\
\hline & $k g / h a$ & $k g / h a$ & $\mathrm{~kg} / \mathrm{ha}$ & $k g / h a$ \\
\hline $\mid A C-5 \ldots \ldots, \ldots$ & 1950 & 470 & 950 & 1123 \\
\hline Sonora-63 ... & 805 & 930 & $\quad 1830$ & 1188 \\
\hline S-12 $\ldots \ldots \ldots \ldots$ & 1755 & 1933 & 660 & 1451 \\
\hline Pioneiro $\ldots \ldots \ldots$ & 610 & 418 & 640 & 556 \\
\hline IRN-526-63 .. & 1490 & 993 & 1660 & 1382 \\
\hline LA-1434. & 1100 & 870 & 1680 & 1217 \\
\hline Pitic-62 ..... & 305 & 1720 & 1280 & 1102 \\
\hline LA-1491 .. & 465 & 1018 & 1080 & 856 \\
\hline IRN-152-63 & 1410 & 680 & 1940 & 1343 \\
\hline BH-1146 $\ldots \ldots$ & 1744 & 803 & 1540 & 1362 \\
\hline$F \ldots \ldots \ldots \ldots$ & $57,38 * *$ & $11,79 * *$ & $16,24^{* *}$ & \\
\hline C.V. $\% \ldots \ldots$ & 13,41 & 29,09 & 17,70 & \\
\hline d.m.s. $(5 \%) \ldots$ & 381 & 698 & 573 & \\
\hline
\end{tabular}

Considerando o ensaio instalado em Guatapará, verificou-se que os cultivares S-12 e Pitic-62 apresentaram as melhores produções, diferindo ao nível de $5 \%$ dos demais cultivares estudados nessas condiçōes.

O cultivar IRN-152-63 foi o mais produtivo em Pindamonhangaba, produzindo em média $1940 \mathrm{~kg} / \mathrm{ha}$, porém não diferindo estatisticamente dos cultivares Sonora-63, LA-1434, IRN-526-63 e BH-1146.

Os resultados da análise estatística conjunta dos três experimentos (qu\&dro 2) mostram que não houve efeito significativo para cultivares e locais, porém a interação cultivares $x$ locais foi altamente significativa ao nivel de $1 \%$ pelo teste $\mathrm{F}$. Verifica-se, portanto, que o corportamento relativo dos cultivares varia significativamente de una localidade para outra. 
QUADRO 2. - Análise da variăncia conjunta dos dados de produção de trigo em grảos ( $\mathrm{kg} / \mathrm{ha})$, obtidos em três ensaios de cultivares instalados, em 1971 , em Mococa, Pindamonhangaba e Guatapará, Sāo Paulo

\begin{tabular}{l|r|r|r|r}
\hline \multicolumn{1}{c|}{ Fonte de variação } & S.Q. & G.L. & Q.M. & \multicolumn{1}{|c}{ F } \\
\hline Cultivar $\ldots \ldots \ldots \ldots \ldots$ & 8098370 & 9 & 899819 & 0,76 \\
Local $\ldots \ldots \ldots \ldots \ldots \ldots$ & 2370810 & 2 & 1185405 & 1,01 \\
Cultivar x Local ...... & 21224940 & 18 & 1179163 & $21,90^{* *}$ \\
Residuo médio $\ldots . . . \ldots$ & & 81 & 53833 & \\
\hline
\end{tabular}

Entre os cultivares estudados, verificou-se que o Pioneiro não apresentou adaptação às condições de várzea do Estado de São Paulo, sendo na média geral o menos produtivo.

Observando o quadro 3, nota-se que os cultivarse S-12, IAC-5 e Pioneiro apresentaram suscetibilidade ao acamamento; esse fato impossibilitaria que fossem cultivados comercialmente em condições de várzea, pois com doses maiores de nitrogênio e irrigaçăo o problema poderia ser agravado.

Os cultivares mexicanos portadores de fatores para nanismo, tais como Sonora-63, IRN-526-63, LA-1434, Pitic-62, LA-1491 e IRN152-63, foram resistentes ao acamamento e à ferrugem do colmo, apesar de mostrarem sintomas de sensibilidade ao $\mathrm{Al}^{3+}$ existente nos solos estudados.

Todos os cultivares foram suscetiveis a Helminthosporium $s p$; BH-1146 foi altamente suscetível à ferrugem do colmo e da folha.

\section{4 - CONCLUSOOES}

a) Os cultivares IAC-5, S-12, BH-1146 e IRN-526-63 apresentaram boa adaptação e produção em condição de várzea, na Estação Experimental de Mococa.

b) Os cultivares mais produtivos em Guatapará foram $\mathrm{S}-12$ e Pitic-62, diferindo estatisticamente dos demais.

c) Em condições de várzea, no Campo de Pesquisas, em Pindamonhangaba, destacaram-se quanto à produção os cultivares IRN-152- 


\begin{tabular}{|c|c|c|c|c|c|c|c|c|c|c|c|}
\hline 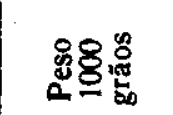 & so & స్త్రి & 今్ & 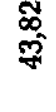 & $\stackrel{\infty}{m}$ & 只 & $\begin{array}{l}\text { N } \\
\text { E̊ }\end{array}$ & की & $\frac{9}{\text { ล }}$ & 5. & $\frac{9}{5}$ \\
\hline 总乐焉 & $\ddot{E}$ & $\stackrel{q}{=}$ & $\stackrel{9}{-}$ & $\underset{-}{Z}$ & $\stackrel{8}{2}$ & 亲 & $\stackrel{m}{=}$ & $\stackrel{\infty}{\rightleftharpoons}$ & $\stackrel{\mathbb{6}}{=}$ & $\stackrel{g}{=}$ & $m_{-}$ \\
\hline 㤎恋 & $\ddot{E}$ & $\frac{\stackrel{n}{m}}{m}$ & $\stackrel{\circ}{\stackrel{8}{s}}$ & $\overline{5}$ & 空 & $\begin{array}{l}\infty \\
\stackrel{\leftrightarrow}{-1}\end{array}$ & $\begin{array}{l}\infty \\
12\end{array}$ & $\frac{ \pm}{m}$ & $\frac{\pi}{\pi}$ & $\stackrel{0}{\text { N }}$ & $\stackrel{n}{3}$ \\
\hline 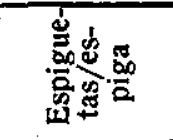 & $\stackrel{*}{*}$ & $\stackrel{m}{n}$ & $\mathbb{E}$ & $\stackrel{\infty}{\infty}$ & $\stackrel{\sim}{2}$ & $\stackrel{\infty}{\infty}$ & $\stackrel{0}{ \pm}$ & $\stackrel{\infty}{=}$ & $\stackrel{\dddot{g}}{\underline{z}}$ & $\stackrel{\dddot{v}}{2}$ & $E$ \\
\hline 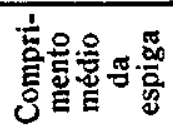 & E & कి & 8 & $\overrightarrow{\text { సे }}$ & 㣽 & $\underset{\infty}{\mathbb{N}}$ & 尺్ & $\underset{\infty}{\mathbb{R}}$ & 8 & 8 & $\stackrel{\mathbb{S}}{-}$ \\
\hline 咅总㤩 & & $\underset{\infty}{\infty}$ & $\mathscr{8}$ & 吅 & 号 & 告 & 先 & $\mathscr{8}$ & $\frac{n}{6}$ & 号 & $\mathscr{D}$ \\
\hline 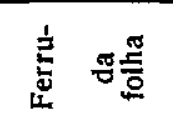 & & $\mathscr{g}$ & 0 & $\stackrel{\varnothing}{\varnothing}$ & g & 孞 & 0 & 0 & 0 & 0 & है \\
\hline 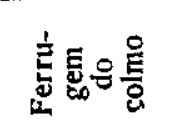 & & $\mathscr{8}$ & 0 & 0 & 0 & 0 & 0 & 0 & 0 & 0 & 8 \\
\hline 䁇。 & & : & $\begin{array}{l}\text { 蓉 } \\
\text { z }\end{array}$ & 鸹 & 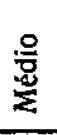 & $\begin{array}{l}\text { s } \\
\text { Z } \\
z\end{array}$ & $\begin{array}{l}\text { 吾 } \\
\text { Z }\end{array}$ & $\begin{array}{l}\text { 胥 } \\
\text { z } \\
\end{array}$ & $\begin{array}{l}\frac{\pi}{7} \\
z\end{array}$ & $\begin{array}{l}\frac{5}{8} \\
\text { 乙 }\end{array}$ & 蒿 \\
\hline 응료 & & 욕 & $\stackrel{ }{\equiv}$ & 요 & $\stackrel{2}{\mathbb{N}}$ & 尺 & తి & $\stackrel{\text { 空 }}{ }$ & $\stackrel{g}{\mathcal{G}}$ & $\stackrel{ }{\underline{\Xi}}$ & శ్ \\
\hline 营 & $E$ & 8 & $\stackrel{18}{8}$ & ह & 5 & $\frac{m}{\sigma}$ & $\stackrel{10}{\infty}$ & $\stackrel{\infty}{\sigma}$ & $\frac{R}{6}$ & $\stackrel{\infty}{\stackrel{\infty}{\sigma}}$ & స్ \\
\hline$\frac{\sqrt[2]{5}}{0}$ & & $\begin{array}{l}\vdots \\
\vdots \\
u \\
\vdots\end{array}$ & 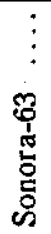 & $\begin{array}{c}\vdots \\
\stackrel{y}{\dot{y}}\end{array}$ & 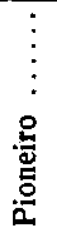 & 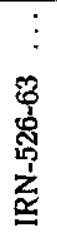 & 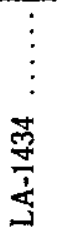 & ֻัญ & $\frac{\dot{5}}{\frac{5}{4}}$ & 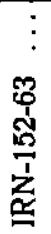 & $\frac{\mathscr{q}}{\frac{1}{1}}$ \\
\hline
\end{tabular}


63, Sonora-63, LA-1434 e IRN-526-63, sendo que somente o cultivar IRN-152-63 diferiu estatisticamente dos cultivares Pitic-62, LA-1491, IAC-5, S-12 e Pioneiro.

d) Os cultivares S-12, IAC-5 e Pioneiro foram suscetiveis ao acamamento, ao passo que os cultivares mexicanos estudados foram resistentes.

e) O cultivar BH-1146 apresentou-se altamente suscetível às ferrugens do colmo e da folha.

f) Todos os cultivares estudados foram suscetíveis a Helminthosporium $s p$.

g) Deverão ser continuadas as pesquisas no sentido de serem criados, através de um programa de melhoramento genético, cultivares adaptados às condiçōes de várzea e portadores de qualidades, tais como produtividade, resistência às moléstias e ao acamamento e tolerância à presença de $\mathrm{Al}^{3+}$ no solo.

\section{BEHAVIOUR OF WHEAT CULTIVARS IN LOW LAND CONDITIONS IN SAO PAULO STATE}

\section{SUM M A R Y}

This paper presents the results of three competition trials of wheat cultivars (Triticum aestivum L.). They were carried out in low land conditions with irrigation, in São Paulo State, Brazil.

The cultivars lAC-5, S-12, BH-1146 and IRN-526-63 were superior on the Mococa Experiment Station. The cultivars $\mathrm{S}-12$ and Pitic-62 gave good results on Jamic Farm, Guatapará. The cultivars IRN-152-63, Sonora-63, LA-1434 and IRN-526-63 showed good performance in Pindamonhangaba.

Besides grain yield, plant height, lodging and resistance to diseases number of kernels and number of spikelets per spike, spike length and number of grain per spikelet were also considered. A detailed description of the pedigree of each cultivar is given in this work.

\section{LITERATURA CITADA}

1. BORLAUG, N. E. Mejoramiento del trigo: su impacto en el abastecimiento mundial de alimentos. México, Centro Internacional de Mejoramiento de Maiz y Trigo, 1969 . $40 \mathrm{p}$.

2. CAMARGO, C. E. O. Ensaios regionais. In: Encontro sobre triticultura. Campinas, CATI, 1971. 28p.

3. Relatórios dos trabalhos desenvolvidos pela Seção de Arroz e Cereais de Inverno do Instituto Agronomico. Campinas, 1969 a 1971. (Não publicados) 
4. CAMARGO, C. E. O.; ALCOVER, M. \& ISSA, E. Comportamento de cultivares de trigo em condiçôes de sequeiro no Estado de São Paulo. Bragantia 33:43-53, 1974.

5. INSTITUTO INDIANO DE PESQUISAS AGRICOLAS. Cinco anos de pesquisas sobre trigo-anăo. Banco do Brasil S.A., 1968. $70 \mathrm{p}$.

6. KERRIDGE, P. C. KRONSTAD, W. E. Evidence of genetic resistance to aluminum toxicity in wheat (Triticum aestivum Vill., Host). Agron. J. $60: 710-711,1968$. 